\title{
Linx
}

LinX Revue des linguistes de l'université Paris X Nanterre

45 | 2001

Invariants et variables dans les langues. Études typologiques

\section{La nature des invariants interlangues}

\section{Gilbert Lazard}

\section{OpenEdition}

\section{Journals}

Édition électronique

URL : http://journals.openedition.org/linx/712

DOI : $10.4000 /$ linx. 712

ISSN : 2118-9692

Éditeur

Presses universitaires de Paris Nanterre

\section{Édition imprimée}

Date de publication : 1 décembre 2001

Pagination : 9-17

ISSN : 0246-8743

\section{Référence électronique}

Gilbert Lazard, «La nature des invariants interlangues », Linx [En ligne], 45 | 2001, mis en ligne le 18 juin 2012, consulté le 20 avril 2019. URL : http://journals.openedition.org/linx/712 ; DOI : 10.4000/ linx.712

Ce document a été généré automatiquement le 20 avril 2019

Département de Sciences du langage, Université Paris Ouest 


\title{
La nature des invariants interlangues
}

\author{
Gilbert Lazard
}

\section{La quête des invariants}

\subsection{Existence des invariants}

1 La croyance à l'existence d'invariants (ou universaux) du langage est spontanée. Si ce n'était le cas, comment des voyageurs, missionnaires et autres curieux de langues auraient-ils si souvent et si longtemps décrit celles qu'ils rencontraient dans les termes de la grammaire latine ou d'autres langues européennes, y trouvant des noms et des verbes, des déclinaisons, des temps, des pronoms, des adverbes, etc. ? Ils voyaient les peuples exotiques qu'ils fréquentaient échanger des informations par des moyens semblables à ceux qu'utilisent nos sociétés, y reconnaissaient des formes de la faculté humaine du langage, apprenaient progressivement à s'en servir eux-mêmes avec succès, et $\mathrm{y}$ identifiaient tout naturellement les mêmes catégories que dans leurs propres langues. Ils n'avaient pas tort, puisque les grammaires qu'ils ont rédigées permettent bel et bien d'accéder à une certaine maîtrise des langues en question.

2 Nous savons bien aujourd'hui qu'elles ne sont pas parfaitement adéquates, et même qu'il s'en faut de beaucoup. Cette expérience n'en reste pas moins la preuve que les langues ont beaucoup en commun. La question est de savoir quoi exactement. C'est une question difficile, sur laquelle s'échinent les linguistes qui s'adonnent à la quête des universaux empiriques du langage. Leur objectif est en somme de donner une forme précise à des traits communs sentis confusément et qui ne se manifestent qu'à travers des formes d'une variété pratiquement infinie.

3 Les recherches de ce type sont aujourd'hui à la mode. Elles bénéficient de l'accroissement considérable de la documentation sur les langues du monde, et elles occupent bon nombre de linguistes. Pour nous en tenir au domaine des structures grammaticales, c'est 
vers le milieu du $\mathrm{XX}^{\mathrm{e}}$ siècle que la quête des universaux s'est affirmée comme une branche originale de la linguistique. Hjelmslev et Benveniste, linguistes saussuriens, ont fait oeuvre de pionniers, l'un dans sa tentative de fonder une linguistique scientifique sur la base de la typologie, l'autre dans une série d'études de "syntaxe générale " (Lazard 1997a). Mais c'est surtout Greenberg et ses disciples qui ont donné le branle.

\subsection{Les universaux de Greenberg}

Le point de départ est le colloque de Dobbs Ferry (1961, cf. Greenberg, éd.,1966), où a vu le jour la fameuse liste, établie par Greenberg et souvent évoquée depuis, de quarante-cinq universaux. La plupart de ces propositions concernent l'ordre des mots. Il s'agit de l'ordre des termes principaux, sujet, objet, verbe, dans la proposition, de celui des éléments, noyau et déterminants, dans le syntagme nominal, ainsi que des variations qui peuvent intervenir dans la phrase interrogative, dans des subordonnées, etc.. Je rappelle ici les cinq premières à titre d'exemples :

1. In declarative sentences with nominal subject and object, the dominant order is almost always one in which the subject precedes the object.

2. In languages with prepositions, the genitive almost always follows the governing noun, while in languages with postpositions it almost always precedes.

3. Languages with dominant VSO order are always prepositional.

4. With overwhelmingly greater than chance frequency, languages with normal SOV order are postpositional.

5. If a language has dominant SOV order and the genitive follows the governing noun, then the adjective likewise follows the noun.

D'autres propositions, d'un type différent, concernent la présence ou l'absence de certaines catégories dans certaines conditions. En voici quelques exemples :

29. If a language has inflection, it always has derivation.

34. No language has a trial number unless it has a dual. No language has a dual unless it has a plural.

36. If a language has the category of gender, it always has the category of number.

43. If a language has gender categories in the noun, it has gender categories in the pronoun.

6 Toutes ces propositions posent une certaine relation entre des éléments du langage, soit relation d'ordre, soit implication de présence ou d'absence. Par exception, le $\mathrm{n}^{\circ} 42$ pose une propriété comme commune à toutes les langues :

42. All languages have pronominal categories involving at least three persons and two numbers.

\section{3. Critique}

Greenberg a eu le grand mérite de lancer le mouvement et l'audace d'établir d'emblée quarante-cinq propositions présumées représenter des propriétés communes à toutes les langues, ou majoritairement présentes, dans des conditions définies. Elles sont fondées sur l'examen d'un échantillon de trente langues (Greenberg, in Greenberg, éd. : 74-75) et aussi sur la riche expérience de l'auteur : " on some questions I have gone well outside the sample » (ibid.). Mais sont-ce vraiment des invariants?

On observe d'abord que certaines de ces propositions ne se présentent pas comme telles. Par exemple, dans le $n^{\circ} 1$, cité ci-dessus, il est dit que le sujet précède " presque toujours " l'objet. C'est une généralisation qui ne prétend pas s'étendre pas à la totalité des langues, 
mais seulement à la grande majorité d'entre elles. Sous une forme différente,

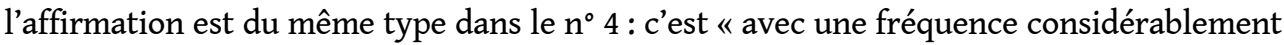
plus grande que celle qui résulterait du hasard ", non dans tous les cas, que les langues SOV sont prépositionnelles. Des formules semblables se trouvent dans diverses propositions, p. ex., $\mathrm{n}^{\text {os }} 9,17,39$, alors que d'autres affirment que telle relation se trouve «toujours » (always ou all languages), p. ex., $\mathrm{n}^{\mathrm{os}} 12,14,15$, ou que la situation inverse ne se trouve «jamais» (never), p. ex., $n^{\circ s} 12$, 37. Greenberg fait donc la distinction entre les traits qu'il pose comme des universaux absolus, de vrais invariants, et les cas où la propriété en question est seulement d'une grande généralité. Ses quarante-cinq propositions, dont beaucoup ont la forme d'une implication ( «si A, alors B »), expriment donc, selon le cas, soit une solidarité entre deux propriétés, soit seulement une affinité.

Une critique plus grave porte sur la nature des notions mises en cause. Poser que le sujet précède l'objet, ou qu'un adjectif précède (ou suit) le nom qu'il qualifie, ou que l'existence de la flexion implique celle de la dérivation, ou que le cas zéro marque prioritairement le sujet du verbe intransitif, etc., implique que l'on sait, en perspective interlinguistique, ce que c'est qu'un sujet, un objet, un adjectif, un cas, un verbe intransitif (et un verbe transitif), comment se distinguent et s'opposent flexion et dérivation, etc. Or, ces notions sont litigieuses. Tout le monde comprend, ou croit comprendre, à peu près de quoi il s'agit, mais il n'y en a pas, en général, de définitions précises. Ce halo d'approximation qui entoure les notions en question enveloppe aussi inévitablement les généralités qui en font état.

10 Greenberg était bien conscient de cette difficulté. Il écrit dans son introduction (1966: 74) : «It is here assumed, among other things, that all languages have subject-predicate constructions, differentiated word-classes, and genitive constructions, to mention but a few. I fully realize that in identifying such phenomena in languages of differing structure, one is basically employing semantic criteria. There are probably formal similarities which permit us to equate such phenomena in different languages. " Il faudrait donc analyser ces critères sémantiques et leur chercher des corrélats formels ${ }^{1}$. Mais c'est là une vaste entreprise. S'y engager, c'était, pour l'auteur, s'écarter de la recherche des universaux empiriques, qui lui paraissait plus urgente. D'ailleurs, ajoute-t-il, « In fact there was never any real doubt in the languages treated about such matters. »

Il est permis de n'être pas de son avis sur ce dernier point. Il n'est pas évident que l'on puisse facilement, en tout langage, identifier sans ambiguïté un terme sujet ou distinguer flexion et dérivation. Mais il est clair que Greenberg a préféré saisir la matière massivement, accepter (peut-être provisoirement ${ }^{2}$ ) les notions traditionnelles avec ce qu'elles comportent d'approximatif, de façon à dégager une série assez importante de caractéristiques qui, à première vue, semblent être soit communes à toutes les langues soit présentes dans la plupart. Il a voulu en somme déblayer le terrain et opérer une première mise en place relativement sommaire. Ce travail s'est révélé fécond, puisqu'il a suscité beaucoup de travaux depuis quarante ans. Mais on peut penser qu'il est temps d'affiner la recherche. 


\section{Les bases de la quête}

\subsection{Catégories}

la liste de Greenberg et dans beaucoup de travaux ultérieurs, dérivent historiquement de celles qui se sont fait jour dans la grammaire des langues occidentales classiques et de quelques langues modernes d'Europe. Elles y sont d'emblée, sans critique, utilisées comme des catégories interlangues. De là leur insuffisance. Le problème qu'elles posent n'est autre que celui de la comparaison des langues, que j'ai évoqué ailleurs (Lazard 1999a et $b$ ).

uis la naissance du structuralisme, on sait bien que les catégories des langues différentes sont toujours différentes. Elles peuvent se ressembler, mais ne coïncident jamais entièrement. Les notions traditionnelles comme celles de sujet, objet, parfait, passif, moyen, perfectif, subjonctif, etc., peuvent prendre un sens précis lorsqu'elles sont appliquées à telle langue particulière, mais, en tant de catégories générales applicables à n'importe quelle langue, elles sont mal définies. Elles se fondent sur la constatation, ou le sentiment, que dans diverses langues, voire de nombreuses langues, on peut identifier (et nommer de même) des catégories qui se ressemblent, c'est-à-dire qui ont un noyau sémantique commun et se traduisent souvent mutuellement (Lazard 1992). Mais il y a toujours des différences, c'est-à-dire des emplois dans telle langue qui n'ont pas de parallèle dans telle autre. Les notions traditionnelles ne font que refléter cette coïncidence partielle entre langues diverses : elles sont donc inévitablement vagues. Il n'y a pas de véritables catégories interlangues. Les prétendues catégories interlangues ne sont que des « quasi-catégories » (Lazard $1997 b$ et c).

Il en résulte que les « universaux » implicationnels du type " si une langue a une catégorie $\mathrm{C} 1$, elle a aussi une catégorie $\mathrm{C} 2$ ", ne peuvent avoir de sens précis. Ils signifient seulement «si une langue a une quasi-catégorie QC1, elle a aussi une quasi-catégorie QC2 ». Ils expriment l'aperception d'une certaine relation entre deux notions floues, dont l'identité est seulement sentie. De telles propositions sont intéressantes et suggestives, mais elles ne satisfont pas l'exigence d'une véritable science du langage, dont l'objectif est de convertir en connaissance précise le sentiment confus de ce que les langues ont en commun.

\subsection{Cadres conceptuels}

Comment faire mieux ? Ma thèse est que le linguiste doit se donner au départ des cadres conceptuels qui serviront d'instruments dans la comparaison des langues (Lazard 1999a et b). Ces cadres sont arbitraires, en ce sens qu'ils ne résultent pas d'un raisonnement rigoureux ni d'une observation systématique. Ce sont des définitions que le chercheur choisit librement, sur la base de son intuition, elle-même nourrie par sa connaissance générale du monde et particulièrement par son expérience des langues. A condition d'être précises, elles lui permettent d'échapper au flou des notions traditionnelles, d'élaborer des hypothèses vérifiables et de les confronter aux faits observables.

La fécondité de la démarche dépend du cadre conceptuel choisi ; sa rigueur et la précision des résultats dépendent de la méthode d'exploration des données de langues. Si, comme dans 
l'exemple ci-dessus, le point de départ est sémantique, le premier pas consiste à "descendre" du sémantique au morphosyntaxique, du contenu à la forme, en l'occurrence de la notion d'action prototypique à la CBM, selon la voie onomasiologique. Mais la suite consiste à confronter d'autres constructions à la CBM : elle se situe toute sur le plan des formes, c'est-à-dire des seuls phénomènes qui soient observables directement. Si l'on veut, dans un second temps (et ce sera souvent nécessaire), faire intervenir les sens convoyés par ces autres constructions, ils ne seront atteints que par la voie sémasiologique, en «montant » de la forme au sens, par les procédés habituels de l'analyse structuraliste. Ainsi sont évitées les incertitudes du recours direct au sens. L'ensemble de la démarche est telle que la part de l'intuition est strictement limitée au choix initial du cadre conceptuel. Les relations invariantes, ou présumées telles, qu'on aura ainsi découvertes (à supposer que le cadre conceptuel initial ait été bien choisi et s'avère fécond) prendront la forme de propositions précises et sans ambiguïté.

\subsection{Desinvariants objectifs}

17 La méthode esquissée ci-dessus, $\$ 2.2$, permet donc en principe d'aboutir à une formulation précise des propositions présentées comme des invariants. Reste à savoir si ces prétendus invariants en sont vraiment, c'est-à-dire si l'on peut les fonder objectivement. Nous formons l'hypothèse que oui.

18 Cette objectivité peut être vérifiée, me semble-t-il, de deux façons. D’une part, les propositions sont confrontées aux données de fait offertes par les langues. Comme elles sont sans ambiguïté, elles peuvent être infirmées par les faits. Si ceux-ci offrent, à une proposition donnée, des contre-exemples dont il s'avère impossible de rendre compte par des conditions particulières, la proposition devra être abandonnée comme fausse. La condition posée par Popper qu'une conclusion scientifique doit pouvoir être «falsifiée » (infirmée) est donc respectée.

Par exemple, l'universau greenbergien $n^{\circ} 1^{3}$ n'est guère "falsifiable", parce que ses termes (sujet, objet) ne sont pas définis; en outre, il est souvent délicat de dire quel est, dans une langue donnée, l'« ordre canonique » des éléments (Dryer 1995). En revanche, si l'on raisonne à partir du cadre conceptuel évoqué ci-dessus (\$ 2.2), les deux termes de la CBM sont facilement identifiables en toute langue ; quant à l'ordre canonique, ce sera tout simplement celui des termes dans la CBM. Il sera donc aisé de voir si, dans cette construction, le représentant de l'agent (que nous appelons $\mathrm{X}$ ) précède toujours le représentant du patient (que nous appelons $\mathrm{Y}$ ) ou non ${ }^{4}$. Le même raisonnement s'applique dans le cas de tous les invariants qui mettent en cause l'ordre des termes.

Une autre façon de s'assurer de l'objectivité des invariants découverts est la suivante. Comme les cadres conceptuels initiaux sont arbitrairement choisis, il est toujours possible d'en changer. On peut donc explorer la même matière en prenant pour points de départ des cadres conceptuels différents. S'il apparaitt que, en suivant ainsi des voies différentes, on aboutit à des conclusions semblables, on sera en droit de les regarder comme des résultats objectifs. 


\section{Variétés d'invariants}

\subsection{Typologie des invariants} plus ou moins abstraites: on présume que les plus fondamentales sont aussi les plus abstraites (et les plus difficiles à formuler). Dans le "Memorandum» introductif du colloque de Dobbs-Ferry, Greenberg et coll. (1966: XXI-XXII) en distinguent quatre types : " phonological, grammatical, semantic, and symbolic. In this classification, the first three involve either form without meaning or meaning without form, whereas the last, which is concerned with sound symbolism, involves the connection between the two. »

La tripartition indiquée dans cette dernière phrase paraît plus importante que la quadripartition selon les secteurs de langue ${ }^{6}$. Nous retenons donc la distinction entre les relations selon qu'elles impliquent seulement des formes ou seulement des contenus de sens ou la corrélation entre les deux ${ }^{7}$.

\subsection{Invariants de forme}

La plupart des universaux de la liste de Greenberg sont des invariants de forme ${ }^{8}$. Il en va de même apparemment de nombre de ceux qui figurent dans l'archive de Plank (v. Plank et Filimonova 2000).

Nous avons vu que certains d'entre eux ne se donnent pas pour des invariants absolus, c'est-à-dire sans exception, mais seulement des relations qui se vérifient dans une majorité de langues, et qui s'interprètent comme attestant, non une loi universelle, mais une tendance. En outre, il n'est même pas sûr que ces tendances soient démontrées. Les universaux en question sont encore plus ou moins subjectifs, étant fondés sur un échantillon de langues assez restreint. On a montré, par exemple, sur la base d'un échantillon de 700 langues réparties dans le monde entier, que "First, except for genitives, [...] verb-initial languages do not exhibit any greater tendency than SVO languages to place modifiers after the noun. And, second, with the exception of genitives and relative clauses, OV languages are no more likely to place modifiers before the noun than VO languages are » (Dryer $1998: 301)$.

Finalement, même s'il existe effectivement des tendances générales régissant l'agencement des formes et se reflétant dans des situations plus ou moins nettement majoritaires, on peut se demander s'il est possible de trouver des invariants de forme qui soient véritablement absolus. En effet, toutes les langues sont malléables dans leur forme, et il n'est pas prouvé qu'il y ait une limite à leur malléabilité. Les effets d'aires montrent combien des langues de types radicalement différents peuvent s'influencer mutuellement. Même dans les cas où il existe une tendance puissante à associer tel trait $\mathrm{A}$ à un trait B et à exclure le trait B', il est fort possible que, dans des conditions particulières, par exemple quand une langue minoritaire est soumise à l'influence constante d'une autre langue, elle en vienne finalement à associer B' à $\mathrm{A}$, en dépit de la tendance typologique. On peut donc douter que l'examen des formes seules puisse aboutir à la découverte de véritables invariants. 


\subsection{Invariants de sens} posé comme cadre conceptuel initial la combinaison de deux oppositions, l'une purement aspectuelle entre inaccompli et accompli, l'autre entre non-concomitant et concomitant (avec un repère temporel), l'auteur constate, après exploration d'une série de langues de types divers, que, dans l'évolution des systèmes aspectuels, les créations nouvelles sont toujours des formes exprimant la concomitance et que celles qui disparaissent ou prennent des valeurs modales sont toujours des formes non-concomitantes. Le phénomène peut être décrit comme un mouvement du concomitant au non-concomitant, puis à la disparition de la valeur aspectuelle, le tout corrélatif du renouvellement de l'expression du concomitant. Nous avons affaire ici à une relation diachronique apparemment invariante, qui met en cause une façon probablement universelle de conceptualiser les procès et, en même temps, un processus évolutif sans doute en rapport avec les conditions d'exercice du langage.

\subsection{Invariants de corrélation} fondées sur la corrélation des contenus de sens et des formes qui les expriment.

1) Accord objectal différentiel. L'accord objectal consiste en ce que que la forme verbale porte un affixe (ou un clitique) qui varie en corrélation avec l'objet ${ }^{9}$. Dans certaines langues (langues bantoues, finno-ougriennes, amharique, etc., et aussi, en un sens, langues balkaniques, cf. Lazard 1994 : 232-234, et 1996), cet accord a lieu ou non selon certaines propriétés de l'objet : ces propriétés sont le degré de définitude et le fait d'être thématique ou rhématique. Dans ce cas, c'est toujours l'objet défini et/ou thématique qui appelle l'accord et avec l'objet indéfini et/ou rhématique que le verbe ne s'accorde pas. En outre, si la langue admet une certaine liberté de l'ordre des mots, l'objet défini/ thématique peut se déplacer dans la proposition et s'éloigner du verbe ; l'objet indéfini/ rhématique au contraire tend à rester au voisinage du verbe. On est ainsi amené à distinguer deux types de construction actancielle: un type "bipolaire», où le verbe et l'objet (indéfini/rhématique) forment ensemble un seul «pôle » en contraste avec celui que forme le sujet, et un type " tripolaire ", où l'objet (défini/thématique) forme tout seul un pôle. Ce qu'on peut schématiser ainsi (l'ordre des sigles n'est pas significatif) : (1)

$$
\begin{array}{|l||l|l|}
\hline \mathrm{X}-\mathrm{YV} & \mathrm{X}-\mathrm{Y}-\mathrm{V} \\
\hline
\end{array}
$$

31 3) Transitivité. Plus généralement, on peut vraisemblablement poser comme universel un ensemble assez complexe de relations entre sens et forme, qui se laisse ranger sous la 
rubrique de la transitivité (Lazard 1994 : 247-253, et 1998). Si l'on pose comme repère fixe la notion d'action prototypique et, en toute langue, comme « construction biactancielle majeure » la construction qui sert à l'exprimer, on constate, dans beaucoup de langues, l'existence de constructions biactencielles différentes, dont le contenu se distingue, par un trait ou un autre (ou plusieurs), de l'action prototypique, et dont la forme se rapproche, d'une manière ou d'une autre, de celle de la construction uniactancielle. On est ainsi amené à concevoir la transitivité comme une grandeur graduelle, et on peut construire l'échelle (2), qui est une représentation des degrés de transitivité, depuis le maximum sémantique (action prototypique) et morphosyntaxique (construction biactancielle majeure) jusqu'au minimum ou intransitivité sémantique (procès n'impliquant qu'un participant) et morphosyntaxique (construction uniactancielle). Entre les deux se situent des constructions « intermédiaires ", qui expriment des procès à deux participants qui ne sont pas des actions prototypiques : une même langue peut posséder plusieurs constructions intermédiaires :

\begin{tabular}{lll}
$\begin{array}{l}\text { Action } \\
\text { prototypique à } \\
\text { deux participants }\end{array}$ & $\begin{array}{l}\text { Procès à deux } \\
\text { participants } \neq \text { action } \\
\text { prototypique }\end{array}$ & Procès à un participant \\
\hline $\begin{array}{l}\text { Construction } \\
\text { biactancielle } \\
\text { majeure }\end{array}$ & $\begin{array}{l}\text { Autres constructions } \\
\text { biactancielles }\end{array}$ & Construction uniactancielle \\
\hline
\end{tabular}

Les détails varient d'une langue à l'autre, aussi bien sur le plan sémantique que sur le plan morphosyntaxique. Mais il semble bien que les relations soient partout conformes au schéma (2).

Ces trois relations présumées invariantes engagent à la fois des sens, c'est-à-dire des aspects de la représentation du monde, et des formes qui les expriment, c'est-à-dire des faits proprement linguistiques. Elles n'ont de sens et d'intérêt que par la corrélation que l'on constate entre les uns et les autres. Elles sont sensiblement plus complexes que les universaux le plus communément allégués. Ce ne sont pas de simples relations d'implication entre deux propriétés, mais une certaine distribution de formes en corrélation avec une certaine distribution de sens, et ces distributions sont telles que, variables d'une langue à l'autre, elles n'en manifestent pas moins une régularité générale spécifique. De semblables régularités sont le produit de l'interaction des processus cognitifs et des conditions de l'activité langagière. Elles sont donc enracinées au cœur même du langage, et, comme telles, elles ont de bonnes chances d'être véritablement universelles. 


\section{BIBLIOGRAPHIE}

BOSSONG, Georg (1998) « Le marquage différentiel de l'objet dans les langues d'Europe » in Actance et valence dans les langues de l'Europe, éd. Feuillet, J., Mouton de Gruyter, Berlin-New York.

COHEN, David (1989)L'aspect verbal, Presses Universitaires de France, Paris.

DRYER, Matthew S. (1995) « Frequency and pragmatically unmarked word order ", in Word order in discourse, éd. Downing, P. et Noonan, M., Benjamins, Amsterdam-Philadelphia.

- (1998) « Aspects of word order in the languages of Europe », in Constituent order in the languages of Europe, éd. Siewierska, A., Mouton de Gruyter, Berlin-New York.

GREENBERG, Joseph H. (1966) « Some universals of grammar with particular reference to the order of meaningful elements ", in Universals of language, éd. Greenberg, J., The MIT Press, Cambridge (Mass.)-London, $2^{\mathrm{e}}$ éd..

GREENBERG, Joseph H. et al. (1966) « Memorandum concerning language universals », in Universals of language, éd. Greenberg, J., The MIT Press, Cambridge (Mass.)-London, $2^{\mathrm{e}}$ éd..

LAZARD, Gilbert (1992) «Y a-t-il des catégories interlangagières ? », in Texte, Sätze, Wörter und Moneme, Festschrift für Klaus Heger, éd. Anschütz, S., Heidelberger Orientverlag, Heidelberg.

- (1994) L'actance, Presses Universitaires de France, Paris.

- (1996) «Fonction de l'accord verbe-actant », in Faits de langue 8, 151-160.

- (1997a) « Benveniste et la syntaxe générale », in Emile Benveniste vingt ans après, éd. Arrivé, M. et Normand, C., Université de Paris X, Nanterre, (Numéro spécial de LINX).

- (1997b) «Grandeur et misère de la typologie », rapport en session plénière du XVIe Congrès des linguistes, in Proceedings of the XVIth International Congress of Linguists, Amsterdam, éd. Caron, B., Elsevier Science, Amsterdam (CD-rom).

- (1997c) « La typologie actancielle », in Studi italiani di linguistica teorica e applicata. 26 :2, 205-226.

- (1998) « De la transitivité restreinte à la transitivité généralisée », in La transitivité, éd.

Rousseau, A., Presses Universitaires du Septentrion, Villeneuve d'Ascq.

- (1999a) « La linguistique est-elle une science? », in Bulletin de la Société de linguistique de Paris $94: 1,67-112$.

- (1999b) «Comment comparer l'incomparable? ", in Typologie des langues, universaux linguistiques, Actes du colloque organisé les 27 et 28 novembre 1998, éd. Sörés, A. et MarchelloNizia, C., Université de Paris X, Nanterre, (Numéro spécial de LINX).

- (sous presse) «Le marquage différentiel de l'objet », in Language typology and language universals , éd. Haspelmath et al., de Gruyter, Berlin-New York. .

PLANK, Frans et FILIMONOVA, Elena (2000) « The universals archive : a brief introduction for prospective users », in Sprachtypologie and Universalienforschung 53 :1, 109-123. 


\section{NOTES}

1. La recherche des fondements universels des structures grammaticales est certainement une tâche de très longue haleine. Mais elle fait partie justement de la quête des invariants et elle peut même être considérée comme en formant l'aboutissement idéal. Elle ne peut donc pas en constituer la condition préliminaire. Le point de départ de la recherche, à mon avis, ne peut être que dans des définitions arbitraires, v. ci-dessous, § 2.2. Il paraît donc utopique de rechercher des marques formelles qui définiraient des catégories grammaticales universelles.

2. "The tentative nature of the conclusions set forth here should be evident to the reader. Without much more complete sampling of the world's languages, the absence of exception to most of the universals asserted here cannot be fully assured " (Greenberg 1966 : 73).

3. Je suppose, pour les besoins de la démonstration, qu'il s'agit d'un universau absolu et non, comme il se présente en fait, d'un quasi-universau (almost always).

4. J'emploie le sigle X pour éviter le terme de « sujet ». Je préfère réserver ce nom à tout actant possédant des propriétés «subjectales» (cas zéro, commande de l'accord verbal, commande du réfléchi, etc.). En fait, le terme appelé $\mathrm{X}$ est souvent un sujet dans ce sens, mais pas toujours. D'autre part, il n'y a pas d'inconvénient à considérer $\mathrm{Y}$ comme l'objet prototypique et à le dénommer ainsi.

5. Sauf rare exception, comme l'universau greenbergien $n^{\circ} 43$, cité plus haut.

6. Qui est fort discutable. Comment peut-on distinguer " grammatical » et « sémantique », alors qu'il n'y a pas de grammaire sans sémantique grammaticale?

7. En l'étendant, bien au -delà des symbolismes phoniques, à toutes les relations qui engagent à la fois des signifiants et des signifiés.

8. La citation faite plus haut, $\S 1.3$, implique apparemment un fondement sémantique pour les catégories en cause, mais l'auteur renonce à en donner des définitions. Les universaux apparaissent donc comme des relations de forme entre des unités immédiatement (intuitivement) identifiables.

9. Je laisse ici de côté, pour simplifier, la question très sérieuse de savoir comment définir sujet et objet en interlinguistique.

\section{AUTEUR}

\section{GILBERT LAZARD}

